\title{
Synthesis and Antimicrobial Activity of 1,2,4-Triazoles
}

\author{
R.U.ROY, A. R. DESAI and K.R.DESAI* \\ Department of Chemistry \\ Veer Narmad South Gujarat University, Surat-395007, India.
}

Received 10 November 2004; Accepted 14 December 2004

\begin{abstract}
Several 3-(4'-nitrophenyl)-4-(4"-chloro benzamido)-5-substituted phenyl)4H-1,2,4-triazole (3a-3e) and 3-(4'-nitrophenyl)-4-(4"-methyl benzenesulphonamido)5-substituted phenyl)-4H-1,2,4-triazole (4a-4e) have been synthesised. Representative compounds were screened for antibacterial, antifungal activity. Most of them showed significant antibacterial activity.
\end{abstract}

Key words Synthesis, Antibacterial activity, Antifungal activity, Triazole

\section{Introduction}

Nitrogen based heterocyclic compounds are very important in the field of medicinal chemistry. The present triazoles ${ }^{1}$ were prepared because of its good biological activities. Compounds including a 1,2,4-triazole nucleus ${ }^{2}$ and $4-\mathrm{N}$-substitued derivatives ${ }^{3}$ are known to possess various biological activities. Certain compounds having 1,2,4-triazole nucleus ${ }^{4}$ have been reported as fungicidal, ${ }^{5}$ antimicrobial, ${ }^{6}$ anticonvulsant, ${ }^{7}$ antidepressant, ${ }^{8}$ and antibacterial activities. ${ }^{9}$ Moreover benzamido ${ }^{10}$ and sulphonamido ${ }^{11}$ group are very important to enhance some biological activities like antimalarial, antibacterial, anticancer and antitubercular. ${ }^{12}$

The above mention observation prompted us to synthesis the title compounds with anticipation that the combination of biolabile triazole unit with bioactive sulphonamido compounds may enhance their antimicrobial potency as compared to benzamido derivatives.

\section{Experimental Section}

All melting points were determined in PMP-DM scientific melting point apparatus and are uncorrected. The IR spectra were recorded in $\mathrm{KBr}$ on a "Perkin-Elmer RX 1 FT-IR" Spectrophotometer (serial No. 51448) and ${ }^{1} \mathrm{H}$ NMR spectra were determined with "Perkin Elmer Model-32 NMR spectrometer" at $300 \mathrm{MHz}$ using TMS as internal standard and $\mathrm{CDCl}_{3}$ as solvent.

Preparation of 2-(4'nitrophenyl)-5-(substituted phenyl)-1,3,4-oxadiazole (2a-e) A mixture of 4-nitro benzohydrazide (1) $(0.01 \mathrm{~mole})$ and an aromatic acid (2) $(0.01 \mathrm{~mole})$ in $\mathrm{POCl}_{3}$ $(5 \mathrm{ml})$ was refluxed for 5-6 hr. The reaction mixture was cooled to room temperature and then the contents were poured on to crushed ice and neutralised with bicarbonate solution. The resulting solid was dried and crystallized from ethanol: $v_{\max } 1530(\mathrm{C}=\mathrm{N}), 1490\left(\right.$ Asymmetric $\left.\mathrm{ArNO}_{2}\right)$ and 1347 (symmetric $\left.\mathrm{ArNO}_{2}\right) ; \delta$ ppm $\left(\mathrm{CDCl}_{3}\right)$ 6.96-7.94 (m, 8H, aromatic). 
Preparation of 3-(4'-nitrophenyl)-4-(4"-chloro benzamido)-5-substituted phenyl)-4H-1,2,4-triazole (3a-e)

A mixture of 2-(4'-nitrophenyl)-5-(substituted phenyl)-1,3,4-oxadiazole (2a-e) (0.01mole) and 4-chloro benzohydrazide $(0.01 \mathrm{~mole})$ in dry pyridine $(10 \mathrm{ml})$ was refluxed for $6-8 \mathrm{hr}$. The reaction mixture was cooled to room temperature and then the contents were poured on to crushed ice and neutralised with dilute $\mathrm{HCl}$ solution. The resulting solid was dried and crystallized from glacial aceticacid. Physical and characterization data of compound (3a-e) are given in Table-1. IR and ${ }^{1} \mathrm{H}$ NMR spectral data of compound (3a-e) are given in Table-2.

Table-1 Physical and characterization data of compound 3a-4e

\begin{tabular}{|c|c|c|c|c|c|c|}
\hline \multirow{2}{*}{ Compd } & \multirow{2}{*}{$\begin{array}{l}\text { M.P. } \\
{ }^{0} \mathrm{C}\end{array}$} & \multirow{2}{*}{$\begin{array}{l}\text { Yield } \\
(\%)\end{array}$} & \multirow{2}{*}{$\begin{array}{c}\text { M.F. } \\
\text { (Mol. Weight) }\end{array}$} & \multicolumn{3}{|c|}{ Found (Calcd.) (\%) } \\
\hline & & & & $\mathrm{C}$ & $\mathrm{H}$ & $\mathrm{N}$ \\
\hline \multirow{2}{*}{$3-\mathrm{a}$} & \multirow{2}{*}{210} & \multirow{2}{*}{65} & $\mathrm{C}_{21} \mathrm{H}_{14} \mathrm{ClN}_{5} \mathrm{O}_{3}$ & 60.00 & 3.34 & 16.00 \\
\hline & & & 419.820 & $(60.08)$ & $(3.36)$ & $(16.08)$ \\
\hline \multirow{2}{*}{$3-b$} & \multirow{2}{*}{230} & \multirow{2}{*}{60} & $\mathrm{C}_{21} \mathrm{H}_{13} \mathrm{Cl}_{2} \mathrm{~N}_{5} \mathrm{O}_{3}$ & 55.49 & 2.88 & 15.39 \\
\hline & & & 454.265 & $(55.52)$ & $(2.86)$ & $(15.42)$ \\
\hline \multirow{2}{*}{$3-\mathrm{c}$} & \multirow{2}{*}{215} & \multirow{2}{*}{62} & $\mathrm{C}_{22} \mathrm{H}_{16} \mathrm{ClN}_{5} \mathrm{O}_{5}$ & 55.70 & 3.51 & 15.58 \\
\hline & & & 449.846 & $(58.74)$ & $(3.59)$ & $(15.57)$ \\
\hline \multirow{2}{*}{$3-d$} & \multirow{2}{*}{254} & \multirow{2}{*}{58} & $\mathrm{C}_{21} \mathrm{H}_{13} \mathrm{Cl}_{2} \mathrm{~N}_{5} \mathrm{O}_{3}$ & 55.49 & 2.88 & 15.39 \\
\hline & & & 454.265 & $(55.52)$ & $(2.86)$ & $(15.42)$ \\
\hline \multirow{2}{*}{$3-\mathrm{e}$} & \multirow{2}{*}{233} & \multirow{2}{*}{60} & $\mathrm{C}_{22} \mathrm{H}_{16} \mathrm{ClN}_{5} \mathrm{O}_{5}$ & 55.70 & 3.51 & 15.58 \\
\hline & & & 449.846 & $(58.74)$ & $(3.59)$ & $(15.57)$ \\
\hline \multirow{2}{*}{ 4-a } & \multirow{2}{*}{227} & \multirow{2}{*}{66} & $\mathrm{C}_{21} \mathrm{H}_{17} \mathrm{~N}_{5} \mathrm{O}_{4} \mathrm{~S}$ & 57.89 & 3.90 & 16.00 \\
\hline & & & 435.457 & $(57.92)$ & (3.93) & $(16.08)$ \\
\hline \multirow{2}{*}{$4-b$} & \multirow{2}{*}{252} & \multirow{2}{*}{60} & $\mathrm{C}_{21} \mathrm{H}_{16} \mathrm{ClN}_{5} \mathrm{O}_{4} \mathrm{~S}$ & 53.66 & 3.40 & 14.79 \\
\hline & & & 469.902 & $(53.68)$ & $(3.43)$ & $(14.90)$ \\
\hline \multirow{2}{*}{$4-\mathrm{c}$} & \multirow{2}{*}{237} & \multirow{2}{*}{62} & $\mathrm{C}_{22} \mathrm{H}_{19} \mathrm{~N}_{5} \mathrm{O}_{5} \mathrm{~S}$ & 56.70 & 4.09 & 15.00 \\
\hline & & & 465.483 & $(56.77)$ & $(4.11)$ & $(15.05)$ \\
\hline \multirow{2}{*}{$4-d$} & \multirow{2}{*}{280} & \multirow{2}{*}{55} & $\mathrm{C}_{21} \mathrm{H}_{16} \mathrm{ClN}_{5} \mathrm{O}_{4} \mathrm{~S}$ & 53.66 & 3.40 & 14.79 \\
\hline & & & 469.902 & (53.68) & $(3.43)$ & $(14.90)$ \\
\hline \multirow{2}{*}{$4-\mathrm{e}$} & \multirow{2}{*}{271} & \multirow{2}{*}{62} & $\mathrm{C}_{22} \mathrm{H}_{19} \mathrm{~N}_{5} \mathrm{O}_{5} \mathrm{~S}$ & 56.70 & 4.09 & 15.00 \\
\hline & & & 465.483 & (56.77) & (4.11) & $(15.05)$ \\
\hline
\end{tabular}

Preparation of 3-(4-nitrophenyl)-4-(4'"-methyl benzenesulphonamido)-5-substituted phenyl)-4H-1,2,4triazole $(4 a-e)$

A mixture of 2-(4-nitrophenyl)-5-(substituted phenyl)-1,3,4-oxadiazole (2a-e) (0.01mole) and 4methyl benzenesulphohydrazide $(0.01 \mathrm{~mole})$ in dry pyridine $(10 \mathrm{ml})$ was refluxed for $6-8 \mathrm{hr}$. The reaction mixture was cooled to room temperature and then the contents were poured on to crushed ice and neutralised with dilute $\mathrm{HCl}$ solution. The resulting solid was dried and crystallized from glacial 
aceticacid. Physical and characterization data of compound (3a-e) are given in Table-1. IR and ${ }^{1} \mathrm{H}$ NMR spectral data of compound (3a-e) are given in Table-2.

\section{Result and Discussion}

\section{Antibacterial and antifungal activity}

All the compounds (3a-3e \& 4a-4e) from the series were screened for their antibacterial activity against B. subtilis, S. aureus, P. aeruginosa and E. coli. The standard drug used was Furacin and DMF was kept as solvent control. Similarly the antifungal studies were carried out against fungus C. albicans and C. krusei, using Flucanzol as standard. Compounds 3-e, 4-c, 4-d and 4-e showed significant antibacterial activity. Compound 3-a and 4-b showed significant antifungal activity. Activities of compounds $3 \mathrm{a}-3 \mathrm{e}$ and $4 \mathrm{a}-4 \mathrm{e}$ are given in Table-3.

Table-2 IR, ${ }^{1} \mathrm{H}$ NMR spectral data of compound 3a-4e

\begin{tabular}{|c|c|c|}
\hline Compd & $\begin{array}{l}\mathrm{IR}(\mathrm{KBr}) \\
\left(\mathrm{cm}^{-1}\right)\end{array}$ & $\begin{array}{l}\mathrm{NMR}\left(\mathrm{CDCl}_{3}\right) \\
\quad(\delta \mathrm{ppm})\end{array}$ \\
\hline 3-a & $\begin{array}{c}3318(\mathrm{NH}), \\
1630(\mathrm{C}=\mathrm{O} \text { of amide } \mathrm{I}) \\
1525(\mathrm{C}=\mathrm{O} \text { of amide } \mathrm{II}) \\
810(\mathrm{C}-\mathrm{Cl})\end{array}$ & $\begin{array}{c}\text { 6.95-7.94 (m, 13H, aromatic), } \\
9.79(\mathrm{~s}, 1 \mathrm{H}, \mathrm{NH})\end{array}$ \\
\hline 3-b & $\begin{array}{c}3325(\mathrm{NH}), \\
1625(\mathrm{C}=\mathrm{O} \text { of amide } \mathrm{I}) \\
1525(\mathrm{C}=\mathrm{O} \text { of amide } \mathrm{II}) \\
810(\mathrm{C}-\mathrm{Cl})\end{array}$ & $\begin{array}{c}\text { 6.95-7.94 (m, 12H, aromatic), } \\
9.79(\mathrm{~s}, 1 \mathrm{H}, \mathrm{NH})\end{array}$ \\
\hline 3-c & $\begin{array}{c}3318(\mathrm{NH}), \\
1630(\mathrm{C}=\mathrm{O} \text { of amide } \mathrm{I}) \\
1515(\mathrm{C}=\mathrm{O} \text { of amide } \mathrm{II}) \\
810(\mathrm{C}-\mathrm{Cl})\end{array}$ & $\begin{array}{c}3.73\left(\mathrm{~s}, 3 \mathrm{H}, \mathrm{OCH}_{3}\right) \\
\text { 6.94-7.95 (m, } 12 \mathrm{H}, \text { aromatic }) \\
9.79(\mathrm{~s}, 1 \mathrm{H}, \mathrm{NH})\end{array}$ \\
\hline 3-d & $\begin{array}{c}3330(\mathrm{NH}), \\
1635(\mathrm{C}=\mathrm{O} \text { of amide } \mathrm{I}) \\
1525(\mathrm{C}=\mathrm{O} \text { of amide } \mathrm{II}) \\
810(\mathrm{C}-\mathrm{Cl})\end{array}$ & $\begin{array}{c}\text { 6.95-7.94 (m, 12H, aromatic }), \\
9.79(\mathrm{~s}, 1 \mathrm{H}, \mathrm{NH})\end{array}$ \\
\hline 3-e & $\begin{array}{c}3318(\mathrm{NH}), \\
1630(\mathrm{C}=\mathrm{O} \text { of amide } \mathrm{I}) \\
1510(\mathrm{C}=\mathrm{O} \text { of amide } \mathrm{II}) \\
810(\mathrm{C}-\mathrm{Cl})\end{array}$ & $\begin{array}{c}3.73\left(\mathrm{~s}, 3 \mathrm{H}, \mathrm{OCH}_{3}\right) \\
6.94-7.95(\mathrm{~m}, 12 \mathrm{H}, \text { aromatic }) \\
9.79(\mathrm{~s}, 1 \mathrm{H}, \mathrm{NH})\end{array}$ \\
\hline 4-a & $\begin{array}{c}3320(\mathrm{NH}) \\
1355\left(\mathrm{SO}_{2}\right)\end{array}$ & $\begin{array}{c}\text { 6.90-7.90 (m, 13H, aromatic), } \\
9.67(\mathrm{~s}, 1 \mathrm{H}, \mathrm{NH})\end{array}$ \\
\hline 4-b & $\begin{array}{l}3318(\mathrm{NH}) \\
1355\left(\mathrm{SO}_{2}\right) \\
810(\mathrm{C}-\mathrm{Cl})\end{array}$ & $\begin{array}{c}\text { 6.90-7.90 (m, 12H, aromatic }) \\
9.67(\mathrm{~s}, 1 \mathrm{H}, \mathrm{NH})\end{array}$ \\
\hline 4-c & $\begin{array}{l}3315(\mathrm{NH}) \\
1355\left(\mathrm{SO}_{2}\right)\end{array}$ & $\begin{array}{c}3.65\left(\mathrm{~s}, 3 \mathrm{H}, \mathrm{OCH}_{3}\right), \\
\text { 6.90-7.90(m,12H, aromatic), } \\
9.67(\mathrm{~s}, 1 \mathrm{H}, \mathrm{NH})\end{array}$ \\
\hline 4-d & $\begin{array}{l}3320(\mathrm{NH}) \\
1355\left(\mathrm{SO}_{2}\right) \\
810(\mathrm{C}-\mathrm{Cl})\end{array}$ & $\begin{array}{c}\text { 6.90-7.90 (m, 12H, aromatic }) \\
9.67(\mathrm{~s}, 1 \mathrm{H}, \mathrm{NH})\end{array}$ \\
\hline 4-e & $\begin{array}{l}3318(\mathrm{NH}) \\
1355\left(\mathrm{SO}_{2}\right)\end{array}$ & $\begin{array}{c}3.65\left(\mathrm{~s}, 3 \mathrm{H}, \mathrm{OCH}_{3}\right), \\
6.90-7.90(\mathrm{~m}, 12 \mathrm{H}, \text { aromatic }), \\
9.67(\mathrm{~s}, 1 \mathrm{H}, \mathrm{NH})\end{array}$ \\
\hline
\end{tabular}




\section{REACTION SCHEME}<smiles>[R]c1ccc(C(=O)O)cc1</smiles><smiles>[R]CC=CC=C(C=CC)c1nnc(-c2ccc([N+](=O)[O-])cc2)o1</smiles><smiles>NNC(=O)c1ccc(Cl)cc1</smiles>

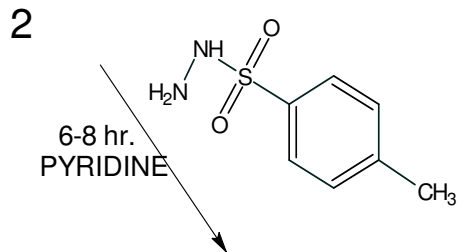<smiles>[R]c1ccc(-c2nnc(-c3ccc([N+](=O)[O-])cc3)n2NC(=O)c2ccc(Cl)cc2)cc1</smiles><smiles>[R]c1ccc(-c2nnc(-c3ccc([N+](=O)[O-])cc3)n2NS(=O)(=O)c2ccc(C)cc2)cc1</smiles>

\begin{tabular}{cccc}
\hline Compound & $\mathrm{R}$ & Compound & $\mathrm{R}$ \\
\hline $3 \mathrm{a}$ & $\mathrm{H}$ & $4 \mathrm{a}$ & $\mathrm{H}$ \\
$3 \mathrm{~b}$ & $o-\mathrm{Cl}$ & $4 \mathrm{~b}$ & $o-\mathrm{Cl}$ \\
$3 \mathrm{c}$ & $o-\mathrm{OCH}_{3}$ & $4 \mathrm{c}$ & $o-\mathrm{OCH}$ \\
$3 \mathrm{~d}$ & $p-\mathrm{Cl}$ & $4 \mathrm{~d}$ & $p-\mathrm{Cl}$ \\
$3 \mathrm{e}$ & $P-\mathrm{OCH}_{3}$ & $4 \mathrm{e}$ & $P-\mathrm{OCH}_{3}$ \\
\hline
\end{tabular}


Table-3. In vitro Antibacterial and Antifungal Activity of compound 3a-4e

\begin{tabular}{|c|c|c|c|c|c|c|}
\hline \multirow{3}{*}{ Compound } & \multicolumn{4}{|c|}{ Antibacterial in $(\mu \mathrm{g} / \mathrm{ml})$} & \multicolumn{2}{|c|}{ Antifungal in $(\mu \mathrm{g} / \mathrm{ml})$} \\
\hline & \multicolumn{2}{|c|}{ Gram positive } & \multicolumn{2}{|c|}{ Gram Negative } & \multirow{2}{*}{$\begin{array}{c}\text { C.albicans } \\
\text { ATCC } 10231\end{array}$} & \multirow{2}{*}{$\begin{array}{c}\text { C.krusei } \\
\text { G03 }\end{array}$} \\
\hline & S.a & B.s & E.c & P.a & & \\
\hline 3-a & 5 & 15 & 10 & 15 & - & 20 \\
\hline 3-b & - & 10 & 0.4 & 5 & 5 & 0.4 \\
\hline 3-c & 15 & - & 15 & 10 & 5 & 15 \\
\hline 3-d & 10 & 5 & 15 & - & - & 15 \\
\hline 3-e & 10 & 25 & 10 & 30 & 10 & - \\
\hline 4-a & 5 & 0.4 & - & 5 & 10 & 0.4 \\
\hline 4-b & - & 0.2 & - & 0.4 & - & 25 \\
\hline 4-c & 30 & 5 & 10 & 15 & - & 5 \\
\hline 4-d & 5 & 20 & 0.4 & 25 & 0.2 & 5 \\
\hline 4-e & 10 & 15 & 30 & 10 & 5 & 0.4 \\
\hline \multicolumn{7}{|c|}{ Zone of inhibition of standard drugs $(\mu \mathrm{g} / \mathrm{ml})$} \\
\hline Furacin & 40 & 45 & 40 & 50 & - & - \\
\hline Flucanzol & - & - & - & - & 40 & 35 \\
\hline
\end{tabular}

\section{Conclusion}

From the above result and discussion about the antimicrobial activity it is conclude that the sulphonamido derivatives are more important in the field of medicinal chemistry with compared to benzamido derivatives which was already anticipated in introductory part.

\section{Acknowledgment}

The authors are thankful to the Head, Department of chemistry, Veer Narmad South Gujarat University, Surat for providing research facilities in the organic research lab and screening of IR Spectra. The authors are also thankful to Quest institute of life science $\mathrm{C} / \mathrm{O}$ Nicolas piramal ltd Mumbai for screening of antibacterial and antifungal screening and CDRI, Lucknow for providing ${ }^{1} \mathrm{H}$ NMR spectra and elemental analysis.

\section{References}

1 Sengupta A K, J Indian Chem Soc 1981, 58, 690.

$2 \quad$ Laddi U V and Desai S R, Indian J Chem 1998, 37, 461.

$3 \quad$ Patel K D and Desai K R, J Indian Chem Soc 2002, 79, 964-965.

4 Tiwari N, Indian J Chem 1993, 32, 399-403

$5 \quad$ Heusach C, Chem Abstract 1980, 92, 181200h.

$6 \quad$ Griffin A D, Chem Abstract 1987, 106, $98120 \mathrm{~h}$.

$7 \quad$ Husain M I, J Indian Chem Soc 1986, 63, 317.

$8 \quad$ Kane J M, Chem Abstract 1987, 107, 115595t.

$9 \quad$ Van R G, Chem Abstract 1979, 91, 753612.

10 Shenoy G G, Indian J Heterocyclic Chem 2001, 10, 197-200.

11 Jain R and Tomar S, J Indian Chem Soc 2000, 77, 44-45.

12 Schmidt L H, Ann Rev Microbial 1969, 23, 427. 


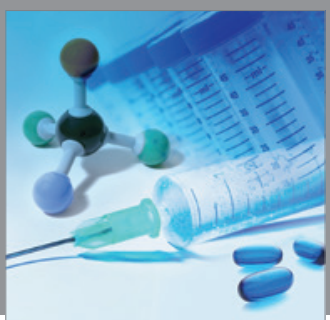

International Journal of

Medicinal Chemistry

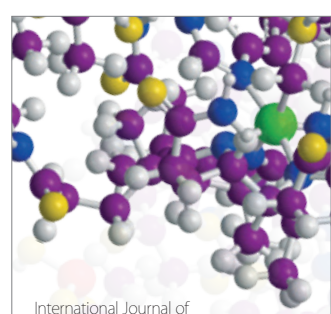

Carbohydrate Chemistry

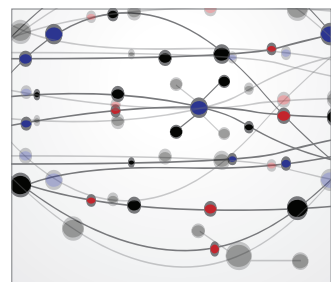

The Scientific World Journal
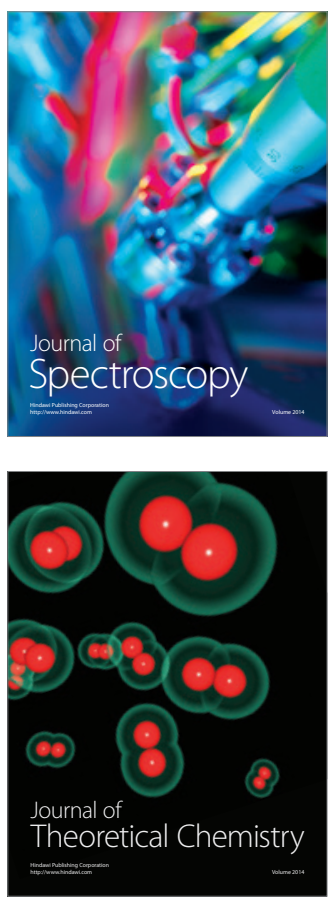
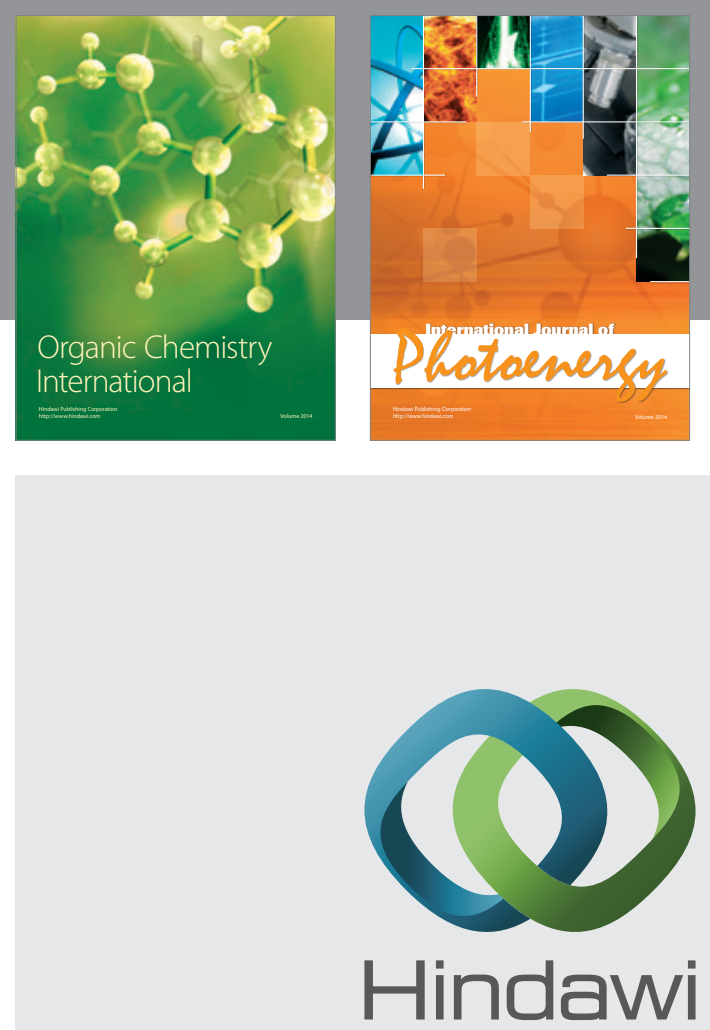

Submit your manuscripts at

http://www.hindawi.com
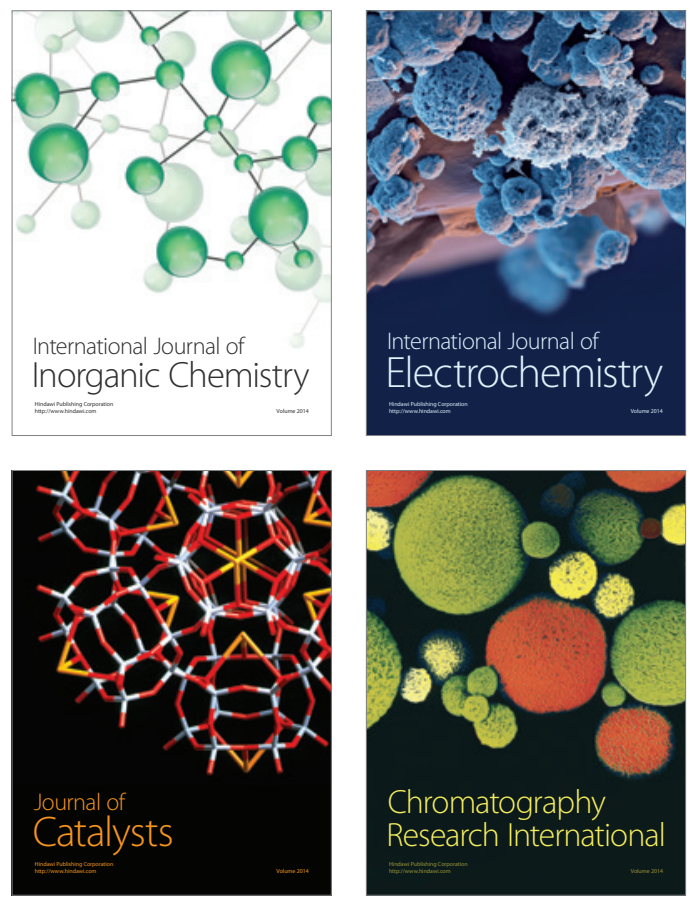
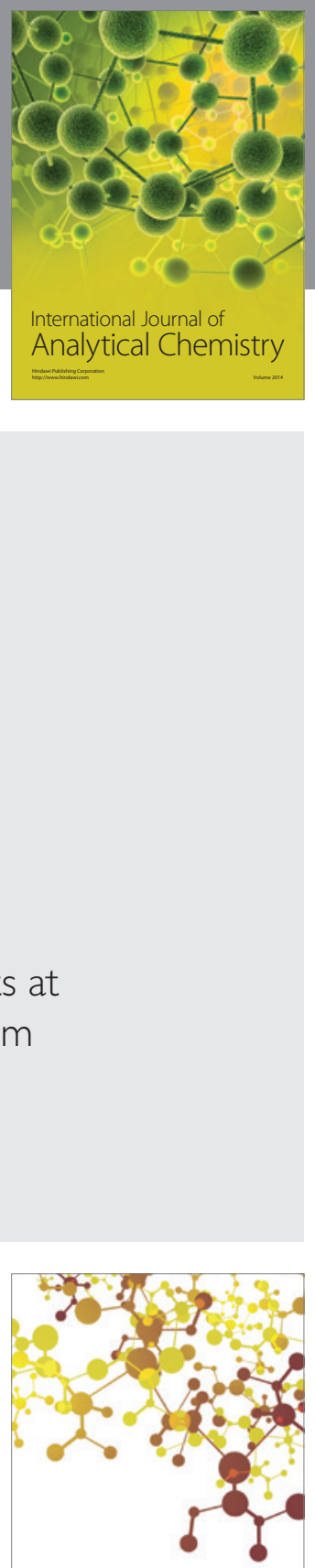

Journal of

Applied Chemistry
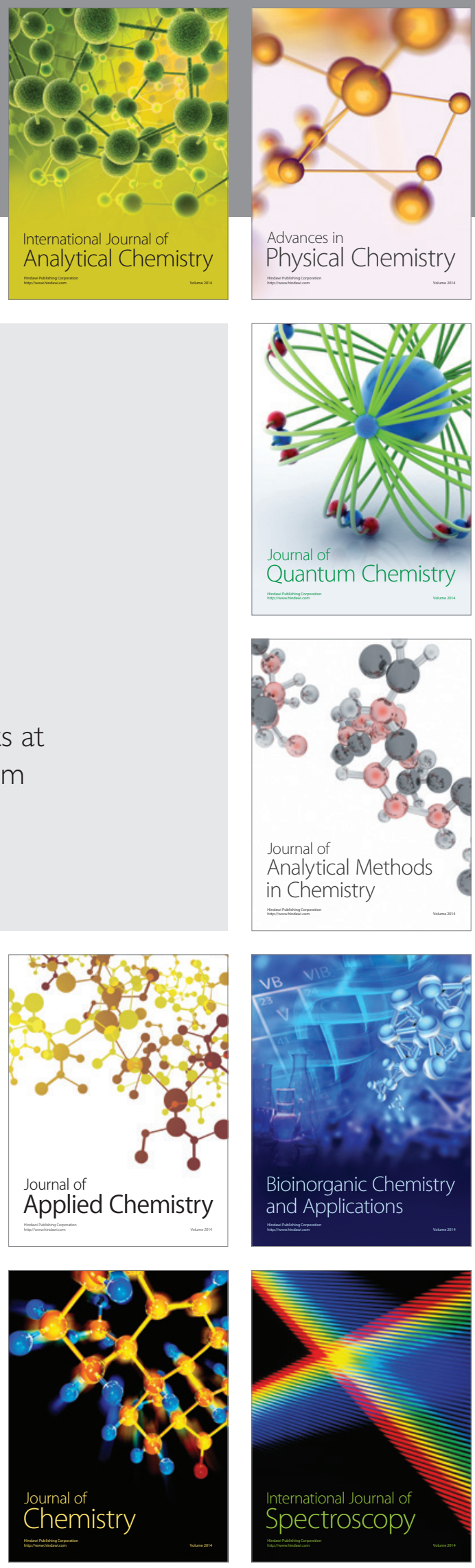